\title{
Vena Cava Inferior Tumor Thrombus Extending to Left Atrium in a Pediatric Patient diagnosed as Wilms Tumor
}

\author{
Pelin Ozcan Kara ${ }^{1 *}$, Zehra Pinar Koc ${ }^{1}$, Elvan Caglar Citak $^{2}$, Yuksel Balci ${ }^{3}$ \\ ${ }^{1}$ Mersin University, Faculty of Medicine, Department of Nuclear Medicine, Mersin, Turkey \\ ${ }^{2}$ Mersin University, Faculty of Medicine, Department of Pediatric Oncology, Mersin, Turkey \\ ${ }^{3}$ Mersin University, Faculty of Medicine, Department of Radiology, Mersin, Turkey
}

*Corresponding Author: Pelin Ozcan Kara, Mersin University, Faculty of Medicine, Department of Nuclear Medicine, Mersin, Turkey,Email: ppelinozcan@gmail.com

\begin{abstract}
Accurate diagnosis of tumor thrombus and distinguishing benign thrombus from tumor thrombus avoid unnecessary anticoagulant treatment of oncological patients and it is important for patient management. In this case report, the role of FDG PET/CT in the demonstration of vena cava inferior tumor thrombus extending to right atrium in a male pediatric patient with Wilms tumor was presented.
\end{abstract}

Keywords: Tumor thrombus; PET/CT; Wilms tumor

\section{INTRODUCTION}

Thrombosis because of venous thrombo embolism (VTE) or tumor thrombosis (TT) is generally more common in oncological patients compared with nononcological patients. Tumor thrombus is a very rare but serious complication of solid tumors. Differantial diagnosis is mostly difficult with radiological modalities such as ultrasonography (US), magnetic resonance imaging (MRI) and diagnostic computed tomography (CT). Distinguishing benign thrombus from tumor thrombus to avoid unnecessary anticoagulant treatment of oncological patients is crucial. In this case report, the role of Fluorodeoxyglucose (FDG)Positron Emission Tomography/ Computed Tomography (PET-CT) in the demonstration of very rare vena cava inferior tumor thrombus in a male pediatric patient with Wilms tumor was presented.

\section{CASE REPORT}

An 8-year-old boy who had a mass in the left kidney and diagnosed with Wilms tumor was sent to Nuclear Medicine Department for the purpose of initial staging with FDG PET/CT imaging. Following 8 hours of fasting, while the patient had a blood glucose level of $91 \mathrm{mg} / \mathrm{dl}, 6$ mCi 18 F-FDG i.v. was injected. After $60 \mathrm{~min}$, images were taken from the calvarium to the soles of the feet in 3D mode for 3 minutes per bed. Obtained images were evaluated after attenuation correction with low dose nondiagnostic CT. On PET-CT imaging, a mass filled the middle-upper part of the left kidney consistent with primary malignancy with increased metabolic activity $($ SUVmax $=15.10)$ and heterogeneous internal structure, reaching a size of about $10 \mathrm{~cm}$, was observed. In addition, tumor thrombus showing evident increased metabolic activity (SUVmax $=10.36)$ in left renal vein lumen, left main iliac artery and vena cava inferior lumen extending to the right atrium was detected. In the whole body imaging, metastatic (SUVmax $=8.7$ ) parenchymal and subpleural nodules with the largest diameter of $3.5 \mathrm{~cm}$ in both lungs, retroperitoneal metastatic lymphadenopathies $($ SUVmax $=7.3)$ and soft tissue dansity in left pulmonary artery lower lobe branch-parahilar area belonging to tumor trombus and/or lymphadenopathy (SUVmax = 10.55) and metastatic hypodense lesion $($ SUVmax $=8.78)$ in L1 vertebral corpus were observed (Figure 1). 


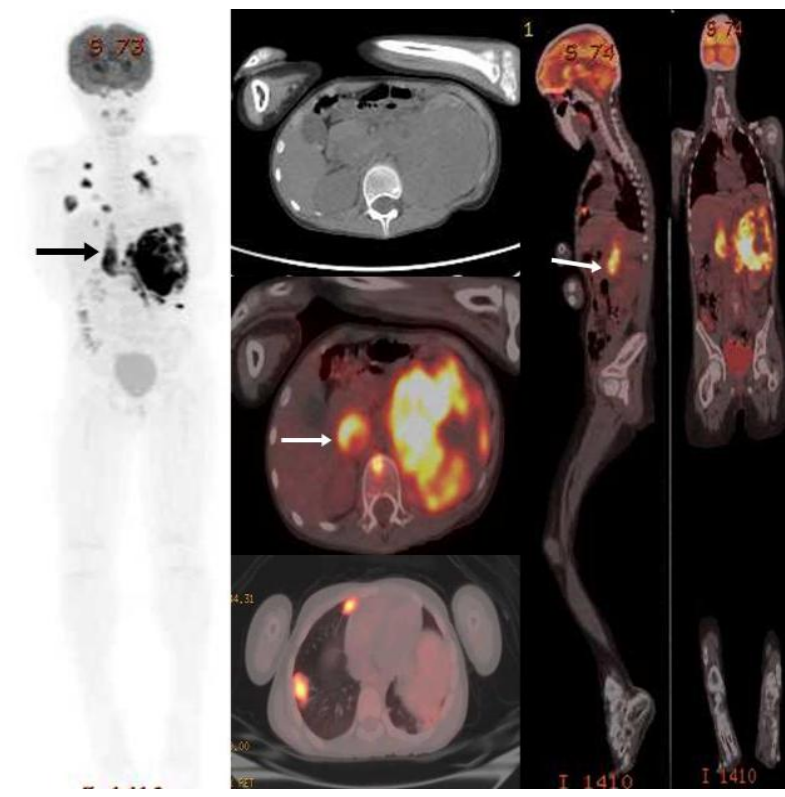

Figure1. MIP (Maximum intensity projection), axial, sagittal and coronal fusion images demontrate dilated VCI linear TT (SUVmax 10.36) with primary left renal tumor (SUVmax 15.10) and widespread metastases in an 8 year old male patient with patological diagnosis of Wilms tumor.

\section{DISCUSSION}

The incidence of thrombus is high in patients with underlying malignancy. This thrombus is mostly venous thromboembolism, but it may rarely be a tumor thrombus. VTE is common in oncologic patients and is treated with anticoagulant agents whereas aggressive treatment approaches are needed in tumor thrombi. Tumor thrombus is a very rare but serious complication of solid tumors. The correct distinction of VTE and TT in oncological patients is critically important for patient management. Accurate diagnosis of tumor thrombus and distinguishing benign thrombus from tumor thrombus provides selection of appropriate therapy. Radiological imaging procedures mostly finds out thrombus insidentally. However, differential diagnosis is difficult with these modalities. FDG PET-CT is a proven procedure in staging, restaging, diagnosis and treatment response in oncological patients. In a recent interesting image report from Sonavane SN et al, the authors reported the imaging findings of a patient with RCC where PET/CT not only ruled out locoregional adenopathy and distant metastases, but also distinguished tumor thrombi from benign thrombi in the same patient (1). In a similar study in the literature for distinguishing benign and malignant tumor thrombus reported $71.4 \%$ sensitivity and specificity values of $\% 90$ with SUVmax cut-off value of 3.63 , while in another study cut-off value has been reported as 2.25 (2$3)$. A recent study of 12 patients on the efficacy of FDG PET/CT for assessing the acuity of DVT found similar results with regards to BT with mean SUVmax of 2.41 (4). In this case, FDG PET-CT imaging was very useful for diagnosis of tumor thrombus with very high metabolic activity in pediatric patient.

\section{Conclusion}

As a conclusion, FDG PET-CT imaging may be useful in oncological both pediatric and adult patients when radiological imaging findings are suspicious for tumor thrombus.

\section{REFERENCES}

[1] Sonavane SN, Malhotra G, Asopa R, Upadhye $\mathrm{T}$. Role of fluorine-18 fluorodeoxyglucose positron emission tomography in a case of renal cell carcinoma to differentiate tumor thrombus from bland thrombus. Indian J Nucl Med. 2015 Oct-Dec;30(4):355-7.

[2] Sharma P, Kumar R, Singh H, Jeph S, Patnecha M, Reddy RM, Naswa N, Bal C, Malhotra A. Imaging thrombus in cancer patients with FDG PET-CT. Jpn J Radiol. 2012 Feb;30(2): 95-104.

[3] Lee EY, Khong PL. The value of 18FFDG PET/contrast-enhanced $\mathrm{CT}$ in detection of tumor thrombus. Clin Nucl Med. 2013 Feb; 38(2):e60-5.

[4] Rondina MT, Lam UT, Pendleton RC, et al. (18)F-FDG PET in the evaluation of acuity of deep vein thrombosis. Clin Nucl Med. 2012; 37:1139-1145.

Citation: Pelin Ozcan Kara, Zehra Pinar Koc, Elvan Caglar Citak, Yuksel Balci. Vena Cava Inferior Tumor Thrombus Extending to Left Atrium in a Pediatric Patient diagnosed as Wilms Tumor. ARC Journal of Clinical Case Reports. 2017; 3(2):11-12. doi:dx.doi.org/10.20431/2455-9806.0302003.

Copyright: () 2017 Authors. This is an open-access article distributed under the terms of the Creative Commons Attribution License, which permits unrestricted use, distribution, and reproduction in any medium, provided the original author and source are credited. 